\title{
A rare cause of reactive arthritis etiology: Bacillus calmette-guerin immunotherapy
}

\author{
Emre Tekgöz ${ }^{1}$, Muhammet Çınar ${ }^{1}$, Sedat Yılmaz ${ }^{1}$ \\ (1) University of Health Sciences, Gulhane Faculty of Medicine, Department of Internal Medicine, Division of \\ Rheumatology, Ankara, Turkey
}

Date submitted:

Aug 07, 2018

Date accepted:

Oct 30, 2018

Online publication date:

March 15, 2019

\section{Corresponding Author:}

Emre Tekgöz

University of Health Sciences,

Gulhane Faculty of Medicine,

Department of Internal Medicine,

Division of Rheumatology,

Ankara, Turkey

dr.emretekgoz@hotmail.com

Keywords: Bacillus Calmette

Guerin, Reactive arthritis,

Spondyloarthritis.

\begin{abstract}
Reactive arthritis belongs to the spondyloarthritis group that manifests up to 6 weeks after a gastrointestinal or genitourinary infection. Usually presents with asymmetric monooligo arthritis on the lower extremity and accompanying extraarticular findings can often be observed on follow-up. Here in, we discussed a case of reactive arthritis developed after intravesical Bacillus Calmette Guerin immunotherapy used as adjuvant treatment for bladder cancer.
\end{abstract}

\section{Introduction}

Reactive arthritis $(\operatorname{ReA})$ is an inflammatory disease which can be presented with oligoarthritis, inflammatory hip and low back pain, extra-articular findings, especially after gastrointestinal and genitourinary system infections (1). In our case, ReA was diagnosed in a patient with the diagnosis of transitional cell carcinoma of the bladder, and treated with mucosal resection and intravesical Bacillus Calmette Guerin (BCG) immunotherapy. In the literature, there are ReA cases due to intravesical BCG immunotherapy used as adjuvant therapy after transurethral mucosal resection of early stage bladder cancer. Our comprehensive overview of this rare disease aims to increase the awareness of ReA which is usually observed in young patients after specific infectious agents, might also be observed at older ages and after atypical infections, or even cancer immunotherapy.

\section{Case}

A 72-year-old male patient was admitted to rheumatology clinic with swelling and pain in the right foot and left 2nd and 3rd fingers. The patient had a diagnosis of transitional cell carcino- ma of the bladder for 6 months. Surgically, mucosal resection was performed and five cycles intravesical BCG immunotherapy was used as adjuvant treatment. At admission, arthritis was detected in the 1-5th metatarsophalangeal joints of the right foot, and dactylitis was detected in the 2-3th finger of the left hand. The patient did not have any other complaints such as inflammatory low back and hip pain. Erythrocyte sedimentation rate and C-reactive protein levels were increased. Rheumatoid factor, anti-nuclear antibody, HLA-B27 were all negative. Mucocutaneous symptoms such as keratoderma blennorrhagicum and circinate balanitis were not detected. There was no symptom or finding consistent with uveitis or conjunctivitis. The patient had not experienced any respiratory tract, gastrointestinal or genitourinary system infectious 6 weeks prior to symptom onset. ReA secondary to intravesical BCG immunotherapy was diagnosed. Non-steroidal anti-inflammatory drugs could not be given because of stage 3 chronic kidney disease. The joint complaints of the patient relieved with the $0.3 \mathrm{mg} / \mathrm{kg}$ prednisolone equivalent methylprednisolone therapy. The patient's complaints did not recur with the reduction of the steroid treatment dose and treatment of the patient was discontinued following a 
remission in the first month of treatment.

\section{Discussion}

ReA belongs to the spondyloarthritis group, which can present with inflammatory back pain, oligoarthritis and extra-articular findings especially after bacterial infection in the gastrointestinal tract or urogenital system within 3 to 6 weeks (1). The disease was first described in 1916 by Hans Reiter in a patient presenting with arthritis, urethritis and conjunctivitis. The disease has become more known due to the increasing series of cases following the Shigella infection. The clinical picture accepted as Reiter's syndrome has been classified under the ReA umbrella because of the more descriptive and variable clinical manifestations (2). ReA affects young people aged 20-40 years and may be associated with HLA-B27 allele. Genetic factors are closely associated with ReA. HLA-B27 positivity varies between $30 \%$ and $50 \%$ in studies, and it can be as high as 60 to $80 \%$ in patients with high disease severity. Besides, some studies have suggested that HLA-B27 positivity may also increase the incidence of disease (3-5).

The first symptom is usually acute joint pain, and fatigue, malaise, or fever may accompany. Asymmetric involvement of large joints, especially in the lower extremities are typical. Inflammatory low back and hip pain may also present in $50 \%$ of patients. Although the symptoms of arthritis decline after 4-5 weeks, in two thirds of the patients joint complaints recur within a year $(6,7)$. Mucocutaneous symptoms are present in up to $50 \%$ of patients. Cutaneous symptoms like psoriasiform eruptions can be observed before or after joint symptoms. Characteristic cutaneous findings are keratoderma blennorrhagicum and circinate balanitis. Additional extra-articular findings are eye, cardiovascular, renal, genitourinary, gastrointestinal, nervous system involvements which are less frequently seen (8). In our case, our patient who underwent mucosal resection and BCG immunotherapy for bladder transitional cell carcinoma developed arthritis in the foot joints and dactylitis in the hand fingers one month after the 5th dose immunotherapy treatment. Even though $\operatorname{ReA}$ is seen at earlier ages, as in our case it can occur at any age after a possible infectious agent. In our case the possible infectious agent could not be detected in the serological tests. After the clinical findings of the patient relieved, glucocorticoid therapy was reduced and the patient's joint findings did not occur within the following one year.

Pathogenesis may be due to excessive immune response to infectious and accumulation of antigens in synovium. Serological or culture tests of the source of the infection are usually negative when arthritis is detected. Evidence of triggering infection and persistent synovial infection should be shown, although the serology and culture tests are negative. Common infectious agents in the gastrointestinal tract are Yersinia, Salmonella, Shigella, Campylobacter jejuni, while the agents commonly encountered in the urogenital system are Chlamydia trachomatis, Neisseria gonorrhoea, Mycoplasma genitalium, Ureaplasma urealyticum. Many factors influence the virulence of microorganisms in the pathogenesis of ReA. These are pathogenic variations, the host's immune system and environmental factors (9). It should be kept in the mind that upper respiratory tract and other rare infectious agents, can take part in the pathogenesis of $\operatorname{ReA}$.

In non-invasive bladder cancer cases, intravesical treatments constitute the main treatment method. BCG vaccine developed from Mycobacterium bovis is used as intravesical immunother- apy in this stage. Intravesical BCG immunotherapy is linked to bladder mucosa and tumor cells. Thus, it increases MHC-II and cytokine secretion from the cells, causes lymphocyte, NK cell and granulocyte accumulation in the tumor tissue and activates the immune system (10). Consequently, activated immune system may be responsible for the development of $\operatorname{ReA}$, as it is seen in our case.

\section{Conclusion}

$\operatorname{ReA}$ is one of the rare complications of intravesical BCG immunotherapy, and should be kept in mind in patients with presenting with arthralgia and/or arthritis after BCG immunotherapy. As in our case, clinical findings usually well controlled with discontinuation of the immunotherapy; besides nonsteroidal anti-inflammatory drugs, and glucocorticoids can be used for symptomatic therapy. Only a small portion of patients develops a chronic process which requires DMARDs therapy.

Acknowledgment: ET: Conception, Design, Data Collection, Writer; MC: Supervision, Materials, Analysis and Interpretation, Writer; SY: Supervision, Writer, Literature Review, Critical Review

Conflict of Interest: The authors declared they do not have anything to disclose regarding conflict of interest with respect to this manuscript.

\section{References}

1. Parker CT, Thomas D. Reiter's syndrome and reactive arthritis. J Am Osteopath 2000; 100(2):101-104.

2. Stavropoulos PG, Soura E, Kanelleas A, et al. Reactive arthritis. J Eur Acad Dermatol Venereol 2015; 29(3): 415-424.

3. Hannu T, Mattila L, Siitonen A, Leirisalo-Repo M. Reactive arthritis attributable to Shigella infection: a clinical and epidemiological nationwide study. Annals of the rheumatic diseases 2005; 64(4): 594-598.

4. Aho K, Ahvonen P, Lassus A, Sievers K, Tiilikainen A. HL-A antigen 27 and reactive arthritis. The Lancet 1973; 302(7821): 157.

5. Kim PS, Klausmeier TL, Orr DP. Reactive arthritis: a review. Journal of Adolescent Health 2009; 44(4): 309-315.

6. Colmegna I, Cuchacovich R, Espinoza LR. HLA-B27-associated reactive arthritis: pathogenetic and clinical considerations. Clin Microbiol Rev 2004; 17(2): 348-369.

7. Wu IB, Schwartz RA. Reiter's syndrome: the classic triad and more. J Am Acad Dermatol 2008; 59(1): 113-121.

8. Schneider JM, Matthews JH, Graham BS. Reiter's syndrome. Cutis 2003; 71(3): 198-200.

9. Sieper J. Pathogenesis of reactive arthritis. Curr Rheumatol Rep 2001; 3(5): 412-418.

10. Donin NM, Lenis AT, Holden S, et al. Immunotherapy for the Treatment of Urothelial Carcinoma. J Urol 2017; 197(1):14-22. 